\title{
Hydrodynamic Conditions of Quorum Sensing in Bacteria
}

\author{
Sarangam Majumdar \\ Department of Mathematics, National Institute of Technology, Rourkela, Odisha-769008, India \\ *Corresponding Author: majumdarsarangam@yahoo.in
}

Copyright $(2013$ Horizon Research Publishing All rights reserved.

\begin{abstract}
In this presentation the hydro dynamical condition of quorum sensing in the biofilm growth is investigated using convection diffusion reaction equation and analyzed environmental condition. It is used to analyze the influence of hydrodynamics and structural heterogeneities on mass transfer in this quorum sensing mechanism. The future direction of stochastic model of quorum sensing in bacteria is given.
\end{abstract}

Keywords Quorum Sensing, Biofilm, Quorum Sensing Molecule (QSM), Mathematicalmodelling, Hydrodynamics

\section{Introduction}

Quorum sensing means a cell -cell communication in bacteria, which is use to coordinate the group behaviour based on population density. This process is regulated by the quorum sensing molecule (QSM) and when it is reached to apparent threshold concentration of QSMs then the cells become induced and undergo gene expressions [23, 26].Quorum sensing was originally discovered in the luminescent bacterium Vibrio fischeri. Vibrio fischeri is a marine bacterium which can be found both as free-living organism and as a symbiont in the light producing organ of an animal host, such as the Hawaiian bobtail squid. The host provides a nutrient-rich environment for the bacterium and the bacterium provides light for the host. It was observed that liquid cultures of Vibrio fischeri produced light only when large numbers of bacteria were present. As a free-living organism, Vibrio fisheri exists at low densities and appears to be non-luminescent. At high cell concentrations, the level of the auto-inducer becomes sufficient to induce transcription of the genes that produce the enzyme luciferase, leading to bioluminescence. A single cell is not capable of producing enough luciferase to cause visible luminescence. Using quorum sensing, the cell can save its effort for the time when sufficient similar cells are around, so that their combined action produces a visible glow. The bacteria thus behave differently in the free-living and symbioticstates. It is important for pathogens to co-ordinate their virulence to escape the immune response of the host and establish a successful infection. The luminescence in Vibrio fischeri is controlled by the transcriptional activation of the lux genes. Most quorum sensing signals are small organic molecules or peptides [27]. Although several quorum sensing systems are known, perhaps the two most thoroughly described systems are the acyl-HSL systems of many Gram-negative bacteria and the peptide -based signaling systems of many Gram- positive bacteria $[16,17,18]$. In this context, Ward et al. [1, 3, 23] proposed the mathematical modeling of quorum sensing and anti-quorum sensing in bacteria and the asymptotic analysis is also studied. A multi-phase mathematical model of quorum sensing in a maturing Pseudomonas aeruginosa is developed by Ward et al. [4, 5]. Dockery and Keener introduced a mathematical model for quorum sensing in Pseudomonas aeruginosa using ordinary differential equations and partial differential equations which is a more realistic model than others [15].Müller et al. analyzed thisCell-cell communication by quorum sensing and dimension reduction [24, 25]. Majumdar et al. [27] also studied mathematical model and stability analysis of quorum sensing mechanism.

Quorum sensing bacteria determine their population density by the production, release and monitoring of one or more diffusible quorum sensing molecules [23]. Different kind of bacteria uses different QSM. We are listed few of them here in the Table1.

This bacterial phenomenon is occurring in the microbial communities which are known as biofilm .Bacteria in the biofilm is protected by the EPS matrix from the external stress. Hydrodynamic environment have directly effect on this bacterial talk $[6,7,8,9]$. These two social phenomena exhibited by bacteria quorum sensing and biofilm development are very much connected.The nutrients availability accelerate the quorum sensing mechanism .Increase the flow indicates the convective supply of nutrients is increased that means the growth is increased, thus the quorum sensing is accelerated. QSM are produced and diffused in a biofilm colony into aqueous phase are transported downstream which delays local up regulation. If the QSM concentration of the aqueous phase is higher than in the biofilm colonies, QSM will diffuse into the colony and promote up regulation even cell densities are relatively low [19,21]. In this context many author proposed different mathematical model and tally this model with 
experimental data. Isaac Klapper and Jack Dockery introduced mathematical description of microbial biofilms [22]. Alpkvist et al. [2] studied three-dimensional biofilm model with individual cells and continuum EPS matrix. Chopp et al. [10, 11, 12] analyzed mathematical model of quorum sensing in a growing $P$. aeruginosa biofilm and dependence of quorum sensing on the depth of a growing biofilm.Cogan developed a two-fluid model of biofilm disinfection $[13,14]$.In this paper we want to investigate the environmental conditions like hydrodynamics and nutrients availability, affective mass transfer and conversion process. These factors are very important in the formulation of quorum sensing and the biofilm formation. We are also draw an outline of future perspectives of the stochastic model of quorum sensing and biofilm growth which is very essential to find out the effect of noise in this spatiotemporal phenomena .This mathematical model of quorum sensing mechanism also investigates the origin of this noise.

Table 1. Structures of Some Quorum Sensing Signal Molecules

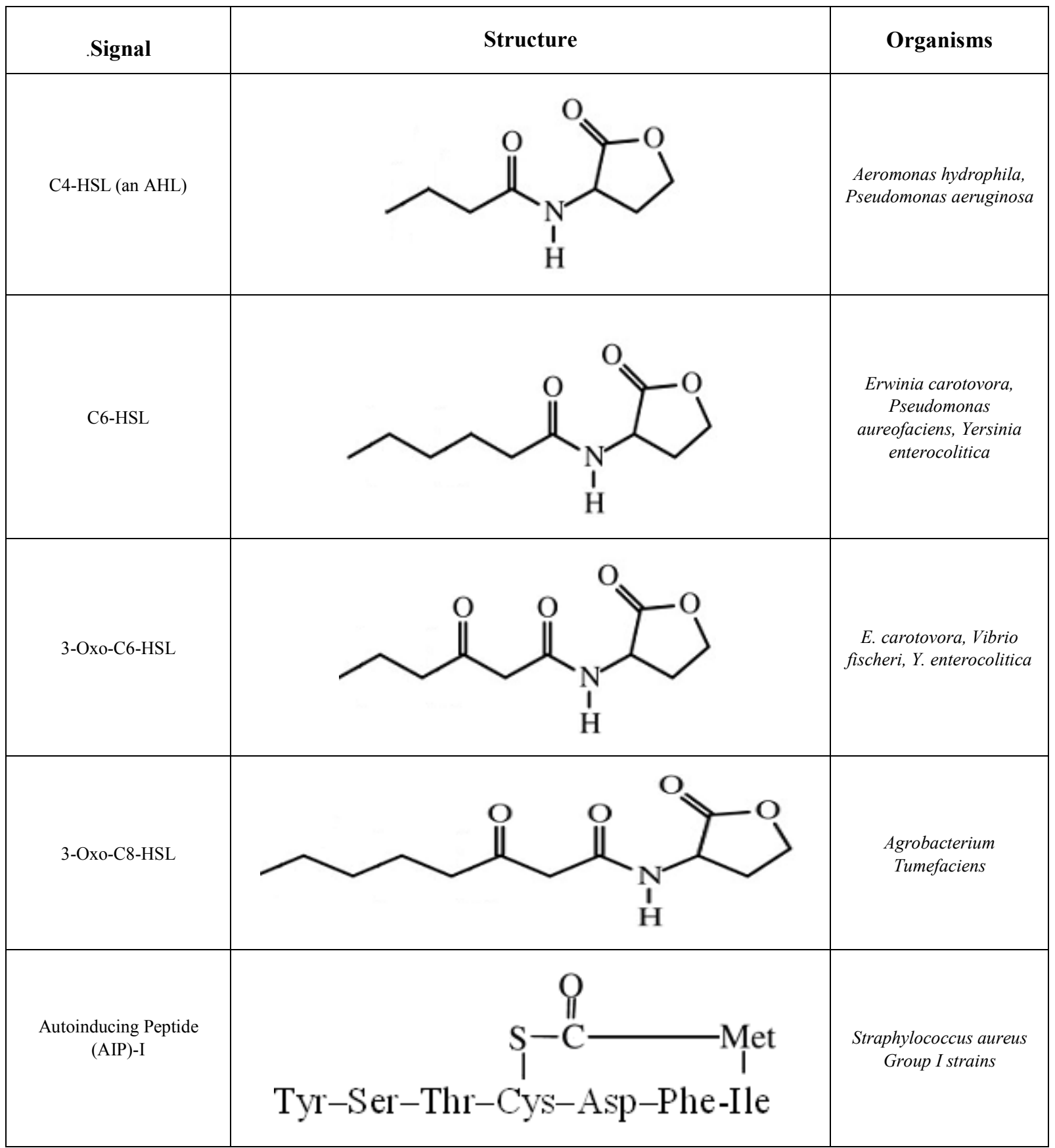



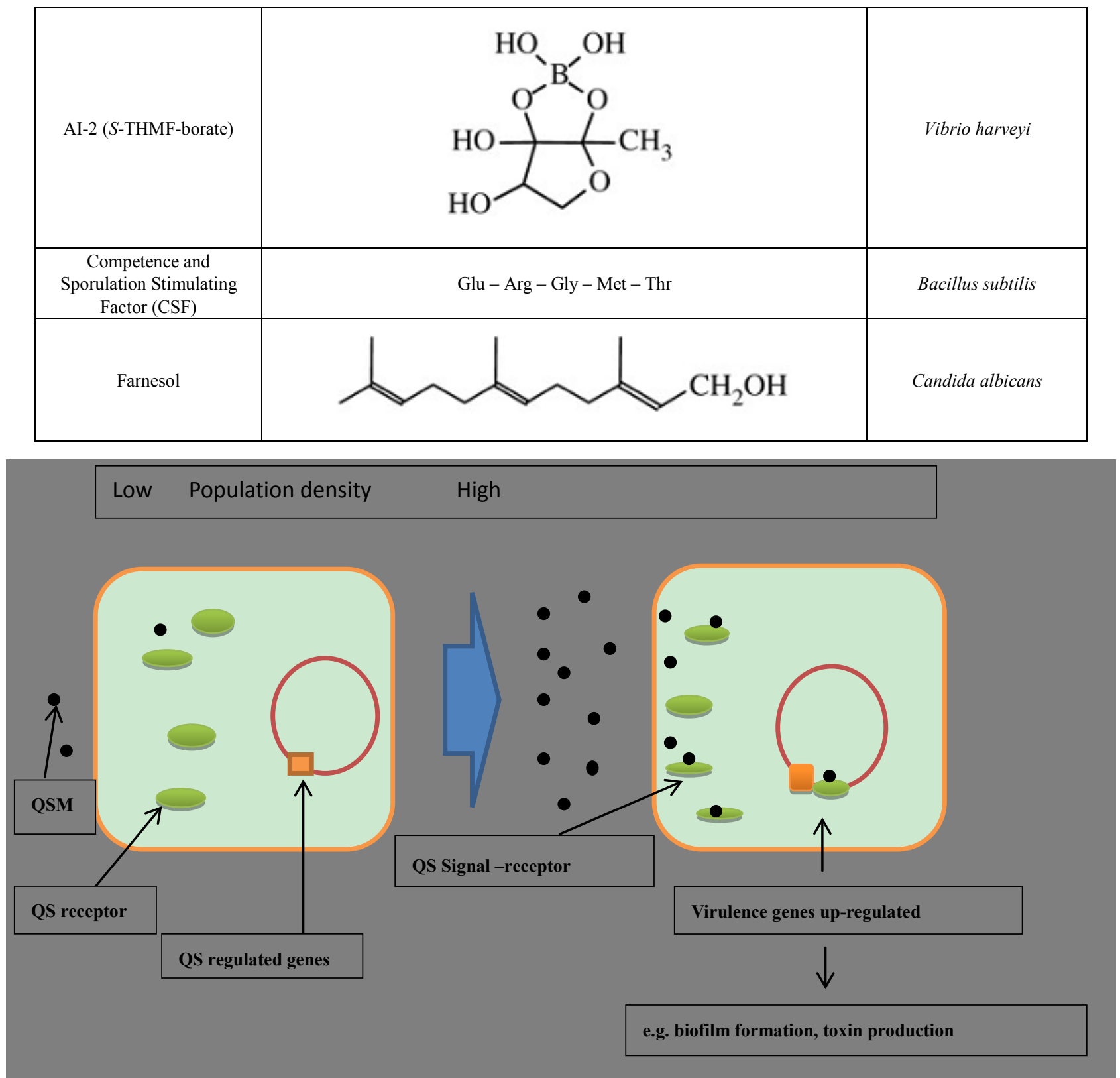

Figure 1. Schematic illustration of quorum sensing system of V.fischeri

\section{Mathematical Model}

Now we introduce a mathematical model which focuses on the hydro dynamical conditions of quorum sensing. Based on the some assumptions that the quorum sensing system not only involving the production of only one QSM but also to the primary QSM of multiple QSM quorum sensing system[23] .Our model is to assume a well-mixed population of cells and convection diffusion reaction equation describes the hydrodynamic environment of the quorum sensing mechanism in the biofilm growth. Let $A$ be the QSM concentration in the fluid, $D$ denote the diffusion coefficient, $\boldsymbol{\vartheta}$ be the average velocity of the QSM and $R$ describes sources of the QSM concentrations.

$$
\frac{\partial A}{\partial t}=\nabla(D \nabla A)-\nabla \cdot\left(\boldsymbol{\vartheta}_{C}\right)+R
$$

with boundary condition $\Omega$.

The hydrodynamic flow field is supported by the incompressible Navier- Stokes equations and the continuity equations for the conservation of liquid mass.

\section{Discussions}

Physical configurations can be described by different characteristic quantities of their fluxes, forces and geometrical shapes. This quorum sensing system is studied by these characteristic ratios.Area enlargement (Ae) refers the geometrical structure of this system .Reynolds number( $\mathrm{Re})$,Schmidt number $(\mathrm{Sc})$, Peclet number $(\mathrm{Pe})$ are refer environmental conditions that means hydrodynamic and the nutrients availability of the systems and Sherwood 
number (Sh)quantifies mass transfer[21].

$\frac{\partial A}{\partial t}=0$ gives the steady state behavior of the quorum sensing system .The equation (1) is directly connected with the equation of continuity where $-D \nabla A$ term comes from the Fick's First law which is known as diffusive flux that arise due to diffusion .on the other hand $(\boldsymbol{\vartheta} c)$ term is known as advective flux .D, $\boldsymbol{\vartheta}, \mathrm{R}$ are vary in space and time that is why we can also consider the Rayleigh-Bénard convection.

When $R=0$ then the equation (1) becomes a stochastic differential equation. $\mathrm{R}=0$ means there is no reaction takes place and this equation describes the motion of QSM where the A describes the probability distribution of QSM is also equivalent to Langevin equation. In the Langevin equation there is a noise term and this noise is regulated the quorum sensing mechanism [20].

\section{Conclusions}

Mass transfer and the environmental conditions are analyzed in this mathematical approach of quorum sensing system. This system is also influenced by the bulk flow. The convection diffusion reaction equation with $\mathrm{R}=0$ is nothing but a stochastic differential equation which is equivalent to Langevin equation. This Langevin equation has potential to describe the source of noise and the noise regulated quorum sensing.

\section{Acknowledgements}

Author would like to thank Prof. Dr. U. Nackenhorsts Prof. Dr. Insa Neuweiler and reviewers for their valuable comments.

\section{REFERENCES}

[1] Anguige K, King JR, Ward JP, Williams P: Mathematical modelling of therapies targeted at bacterial quorum sensing. Mathematical Biosciences, 192:39-83. (2004)

[2] Alpkvist E, Picioreanu C, van Loosdrecht MCM, Heyden A: Three-dimensional biofilm model with individual cells and continuum EPS matrix. Biotechnology and Bioengineering, 94(5):961-979. (2006)

[3] Anguige K, King JR, Ward JP: Modelling antibiotic and anti-quorum sensing treatment of a spatially structured Pseudomonas aeruginosa population. Journal of Mathematical Biology, 51:557-594. (2005).

[4] Anguige K, King JR, Ward JP: A multi-phase mathematical model of quorum sensing in a maturing Pseudomonas aeruginosa biofilm. Mathematical Biosciences, 203(2):240-276. (2006)

[5] Brown, S. P. \& Johnstone, R. A. 2001 Cooperation in the dark: signalling and collective action in quorum-sensing bacteria.
Proc. R. Soc. B 268, 961-965. (2001)

[6] Characklis, W. G. and K. C. Marshall, Biofilms, New York: John Wiley \& Sons, Inc. (1990).

[7] Characklis WG. , Biofilm Development and Destruction. Electric Power Research Institute. Technical report: EPRI CS- 1554, (1980).

[8] Costerton JW, Z Lewandowski, DE Caldwell, DR Korber and HM Lappin- Scott., Microbial biofilms. Annu Rev Microbiol 49: $711-745,(1995)$.

[9] Costerton JW, P Stewart and EP Greenberg., Bacterial biofilms: a common cause of persistent infections. Science 284: 1318-1322, (1999).

[10] Chopp DL: Simulating bacterial biofilms. In Deformable Models. Edited by: Suri JS, Farag AA. Berlin-Heidelberg: Springer; 1-31, (2007).

[11] Chopp DL, Kirisits MJ, Parsek MR, Moran B: A mathematical model of quorum sensing in a growing $\mathrm{P}$. aeruginosa biofilm. Journal of Industrial Microbiology and Biotechnology, 29(6):339-346,( 2002).

[12] Chopp DL, Kirisits MJ, Parsek MR, Moran B: The dependence of quorum sensing on the depth of a growing biofilm. Bulletin of Mathematical Biology, 65(6):1053-1079,(2003).

[13] Cogan NG: A two-fluid model of biofilm disinfection. Bull Math Biol, 70(3):800-819, (2008).

[14] Cogan NG, Keener JP: The role of the biofilm matrix in structural development. IMA Mathematical Medicine and Biology, 21(2):147-166, (2004).

[15] Dockery JD and JP Keener. A mathematical model for quorum sensing in Pseudomonas aeruginosa. Bull Math Biol 63: 95-116. (2001).

[16] Demuth DR, Lamont RJ: Bacterial cell-to-cell communication Cambridge: Cambridge University Press; (2006).

[17] Fuqua, C., S. C. Winans and E. P. Greenberg Census and concensus in bacterial ecosystems: The luxR-luxI family of quorum-sensing transcriptional regulators. Annu. Rev. Microbiol. 50, 727-751. (1996).

[18] Fuqua C and EP Greenberg. Self-perception in bacteria: quorum sensing with acylated homoserine lactones. Curr Opin Microbiol 1: 183-189. (1998).

[19] Frederick MR, Kuttler C, Hense BA, Müller J, Eberl HJ: A mathematical model of quorum sensing in patchy biofilm communities with slow background flow. Canadian Applied Mathematics Quarterly, 18(3):267-298. (2011).

[20] Gardiner C (1996) Handbook of stochastic methods: For physics, chemistry and the natural sciences, Springer Verlag, Berlin.

[21] H.J.Eberl, C.Picioreanu, J.J. Heijnen, M.C.M van Loosdrecht, A three-dimensional numerical study on the correlation of spatial structure, hydrodynamic condition, and mass transfer and conversion in biofilms, Chemical Engineering Science 55(2000),pp. 6209-6222.

[22] Isaac Klapper and Jack Dockery, MathematicalDescription of Microbial Biofilms,Society for Industrial and Applied 
Mathematics: SIAM REVIEW, Vol. 52 No. 2. (2010).

[23] J.P.Ward, J.R. King, A.J. Koerber, P. Williams, J.M.Croft, and R.E. Sockett, Mathematical modelling of quorum sensing in bacteria, IMA Journal of Mathematics Applied in Medicine and Biology 18 (2001), pp. 263-292.

[24] John C March and William E Bentley, Quorum sensing and bacterial cross-talk in biotechnology, Current Opinion in Biotechnology 15, pp. 495-502. (2004).

[25] Müller J, Kuttler C, Hense BA, Rothballer M, Hartmann A:
Cell-cell communication by quorum sensing and dimension reduction. Journal of Mathematical Biology 2006, 53:672-702

[26] Miller MB, Bassler BL, Quorum sensing in bacteria. Annu Rev Microbiol, 55 (2001) pp. 165-199.

[27] Sarangam Majumdar, Suman Datta, Sisir Roy, Mathematicalmodeling of quorum sensing and bioluminescence in bacteria,International Journal of Advances in Applied Sciences (IJAAS),Vol 1,No 3 pp.139-146 (2012). 